\title{
HIV-free survival among breastfed infants born to HIV- positive women in northern Uganda: a facility-based retrospective study.
}

Irene Aguti

Gulu University

Charles Kimbugwe

Gulu University

Patricia Apai

Gulu University

Siraji Munyaga

Gulu University

Richard Nyeko ( $\square$ rnyeko2@gmail.com )

Lira University https://orcid.org/0000-0003-3859-349X

Research article

Keywords: HIV-free survival, Mother-to-child transmission, Breastfeeding, Antiretroviral therapy

Posted Date: February 20th, 2020

DOI: https://doi.org/10.21203/rs.2.13574/v2

License: (c) (1) This work is licensed under a Creative Commons Attribution 4.0 International License. Read Full License

Version of Record: A version of this preprint was published at Pan African Medical Journal on January 1 st, 2020. See the published version at https://doi.org/10.11604/pamj.2020.37.297.22928. 


\section{Abstract}

Background: Without interventions, the risk of mother-to-child transmission of HIV is $25 \%$ during pregnancy, labour and delivery with an additional risk of $5-20 \%$ during the breastfeeding, leading to an overall risk of $45 \%$. The effectiveness of interventions towards prevention of mother-to-child transmission of HIV in any setting is measured by its HIV-free survival rate. We carried out a study to determine the HIV-free survival among breastfed infants enrolled by 2 months of age in a resource-poor setting.

Methods: We conducted a cross-sectional survey of early infant diagnosis registers at two tertiary facilities in northern Uganda and retrospectively reviewed records of exposed infants enrolled from 2014 to 2016. We used simple random sampling and analyzed data using SPSS v16 software package. The chi-square and Student ttests were used to compare factors among HIV-free survivors (HIV-uninfected and alive at 18 months) and those who became HIV-infected or died. Multivariate analysis using logistic regression was used to determine the factors that were independently associated with HIV-free survival. Odds ratios with $95 \%$ confidence interval were used to measure the strength of association between the outcome and predictor variables. P-value $<0.05$ was considered for statistical significance

Results: The mean age at enrolment of the 317 infants was 1.57 (SD 0.23), majority of whom were males $55.5 \%$ (176/317). All except two infants were initiated on Nevirapine prophylaxis. Less than one third, 29.3\% (93/317) of the infants were exclusively breastfed for at least 6 months, while the breastfeeding status of 30 infants could not be ascertained. The overall HIV-free survival rate in the current study was $94.6 \%$ (300/317), while $2.5 \%$ (8/317) were HIV-infected and 2.8\% (9/317) died. Gender and treatment facility were the factors independently significantly associated with HIV-free survival.

Conclusions: Our study estimates that the HIV-free survival rate in the study setting for breastfed infants enrolled by 2 months of age was $94.6 \%$, below the WHO goal of $>95 \%$ HIV-free rate in breastfeeding population for virtual elimination of HIV. A further prospective study would be necessary to assess a more accurate HIV-free survival rate given the limitations of retrospective data.

\section{Introduction}

Even though the incidence of paediatric HIV is falling as a result of an increase in effective methods to prevent mother-to-child transmission of HIV, an estimated 180,000 (110,000-260,000) new paediatric HIV-1 infections occurred in 2017, primarily through mother-to-child transmission, mainly in sub-Saharan Africa (1). In Uganda, a recent survey estimates that 95,000 children aged 0-14 years were living with HIV in 2017 (2), about $90 \%$ of them having acquired the infection through mother-to-child transmission (MTCT). An estimated 7,600 new paediatric HIV infections were registered in the country in 2017, with 3,800 AIDS-related deaths (1).

Without interventions for the prevention of mother-to-child transmission of HIV (PMTCT), the rate of maternal-tochild transmission (MTCT) of HIV during pregnancy and delivery is estimated at $15-25 \%$, and the additional risk through breastfeeding is estimated at $5-20 \%(3,4)$. With interventions for PMTCT, the transmission reduces to $<5 \%$ in the breastfeeding population and to $<2 \%$ in the non-breastfeeding population, making the prevention of mother-to-child transmission of HIV (PMTCT) a major public health approach of reducing the scourge (5). 
Uganda rolled out nationwide implementation of PMTCT option B+ approach in 2013, a policy whereby all HIVinfected pregnant and breastfeeding women were started on combination ART (with lamivudine/tenofovir disoproxil fumarate/efavirenz [3TC/TDF/EFV] as the preferred regimen) for life, regardless of their WHO clinical stage and/or CD4 cell count, following the World Health Organization (WHO) guidance (6). This was later expanded in a 2016 update of the national guidelines (7) following the release of new WHO guidelines in 2015 (8), with the recommendation for lifelong ART for all HIV-positive individuals, including pregnant and breastfeeding women, regardless of clinical stage and CD4 count.

Although there are promising results on program effectiveness for the ever-evolving interventions for elimination of mother-to-child transmission of HIV, data on the long-term effectiveness of these interventions in the study setting is lacking. Furthermore, most HIV-related studies in Uganda have mainly been confined to centres of excellence. This study, therefore, aimed to determine the HIV-free survival rate and associated factors among infants born to HIV positive mothers in northern Uganda in both urban and peri-urban/semi-rural resource-poor upcountry settings, in light of the current efforts to the elimination of mother-to-child transmission of HIV (eMTCT).

\section{Methods}

\section{Study setting}

This study was conducted at two tertiary referral hospitals in northern Uganda - Gulu regional referral hospital (GRRH), a public health facility in an urban setting and St. Mary's hospital Lacor, a private not-for-profit facility in a peri-urban/semi-rural setting. These are the two largest hospitals in the district of Gulu and the sub-region at large, serving the population from the entire northern region and beyond. Gulu district is located approximately 340 kilometres north of Uganda's capital city, Kampala. The population of Gulu district was estimated at 443,733 (9). Both hospitals run antiretroviral therapy (ART) clinics which provide HIV services to the people of Gulu district and beyond. Northern Uganda is a region recovering from over two decades of insurgency with a high burden of HIV (7.2\%) compared to the national prevalence (6.2\%) (2) and access to health services remains a challenge in the district and the region as a whole. High levels of poverty and illiteracy, especially among women, is exacerbated by high prevalence of preventable diseases, including HIV/AIDS.

\section{Study design}

We conducted a cross-sectional survey of early infant diagnosis (EID) registers in May 2019 at St. Mary's Hospital Lacor and Gulu regional referral hospital in Gulu district, northern Uganda. Data for infants born to mothers living with HIV were abstracted from January 2014 to December 2016. Infants who had complete data and documented outcome were included in the study, while those who did not complete the recommended period of follow up and whose outcome was not known, as well as those with missing data, were excluded. The infants with missing data were excluded from this study because we did not have adequate documented information to meaningfully characterize them. Finally, we analyzed the outcome of infants enrolled in care within the recommended 2 months of age. Infants enrolled after 2 months of age were excluded from the final analysis to avoid introducing selection bias since it could be presumed that only surviving infants were likely to enter the groups of infants enrolled at an older age. The minimum sample size was estimated using the formula for crosssectional studies by Leslie Kish (1965) (10) and a standard error of 5\%. 


\section{Determination of infants' HIV status}

The infants final status was based on test results routinely performed using national procedures and algorithms for early infant diagnosis (EID) of HIV. The then Uganda national Consolidated Guidelines for Prevention and Treatment of HIV recommend that all infants born to HIV positive mothers be initiated on Nevirapine prophylaxis at birth according to their risk classifications (7). Infants were then routinely followed up at 6,10 , and 14 weeks, then monthly until 6 months of age and every 3 months until 18 months of age, with a final visit at 24 months. The HIV status of infants were determined using national algorithms for early infant HIV diagnosis, and tested by polymerase chain reaction (PCR) for the detection of viral deoxyribonucleic acid (DNA) using dried blood samples (DBS) collected on filter paper at 4-6 weeks of age or at the earliest time thereafter (7). For infants with an initial negative DNA PCR test result, a second DNA PCR test is performed 6 weeks after cessation of breastfeeding. Infants with an initial HIV DNA PCR positive test result had a confirmatory PCR performed at the time of ART initiation. A rapid HIV antibody testing was conducted at 18 months of age for all infants who test negative at first or second PCR to determine the final HIV status. The national guidelines recommended that all children below the age of 15 years who are confirmed HIV infected are referred for immediate ART initiation regardless of WHO clinical stage or CD4 count/percentage $(7,11)$. Infants with negative DNA PCR test results have an HIV rapid test performed at 18 months of age to determine the final status. The recommendation for maternal ART within the time for which data were collected was based on option $B+$ where pregnant and breastfeeding women were started on 3TC/TDF/EFV as the preferred regimen.

\section{Sampling procedure}

Records in the Early Infant Diagnosis (EID) register for infants enrolled into care from January 2014 to December 2016 were retrospectively reviewed and a sampling frame for all the eligible infants was written down using codes. A simple random sampling method was then used. Simple random sampling is a probability sampling in which each individual has an equal chance of being selected and we believe this was a reasonable approach to get a representative sample. The code numbers were written down on pieces of paper which were folded and put in a container. These were properly mixed and then randomly picked with replacement until the required sample size was reached. Random sampling with replacement was used to allow for an equal chance of being selected into the study for each infant. Replacement involved picking a code at random and putting it back before randomly picking the next. If a code is repeated, it was replaced and another randomly picked until a different code was picked. This process was repeated until the required sample size was reached. Data was collected using data abstraction checklists designed in English and contained variables which were selected based on the study objectives. These were filled in by the researchers with the available recorded data in the source documents of both hospitals. Data was double-checked for completeness and accuracy at the end of each day of data collection, kept in a safe place out of reach of unauthorized persons.

HIV-free survival in this study was defined in line with the WHO definition as "an infant or young child born to a mother living with HIV who remains both HIV uninfected (confirmed negative HIV status) and also alive at 18 months of age" (12).

\section{Data analysis}


Data were entered, cleaned and analysed using Statistical Package for Social Scientists (SPSS) software package (SPSS for Windows, Version 16.0. Chicago, SPSS Inc.). In univariate analysis, categorical variables were summarized as proportions, while continuous variables as means and standard deviations (SD). HIV-free survival rate was calculated as the proportion of study infants who were alive with final HIV-negative status at 18 months, the denominator being all infants enrolled in the study. In the bivariate analysis, the chi-square test (for categorical variables) and Student t-test (for continuous variables) were used to test if the factors among infants with negative HIV status at 18 months were different from those among infants with outcomes other than negative HIV status at 18 months. Odds ratios with 95\% confidence interval (Cl) were used to measure the strength of association between the outcome and predictor variables. Multivariate Cox regression model was used to determine the factors that were independently associated with HIV-free survival. P-value $<0.05$ was considered for statistical significance.

\section{Results}

\section{Description of the study population}

Four hundred twenty-two (422) infants enrolled into care in the two ART facilities between 2014 and 2016 were initially sampled, 317 (75.1\%) of whom were enrolled within the recommended 2 months of age and were analyzed for HIV-free survival. Of the infants, 55.2\% (175/317) were from Gulu regional referral hospital (GRRH) and $44.8 \%$ (142/317) from St. Mary's hospital Lacor (Figure 1). The mean age (in months) of enrolment of the infants into care was 1.57 (SD 0.23) and over half 55.5\% (176/317) of the infants were male. All, except two infants, were initiated on Nevirapine prophylaxis (Table 1), the majority of whom $99.0 \%(312 / 315)$ were initiated within the desired 72 hours of birth. Less than one third, 29.3\% (93/317) of the infants were exclusively breastfed for at least 6 months as recommended by the national guidelines, while the breastfeeding status of 30 infants could not be ascertained (no documentation). Overall, the majority of the infants $86.4 \%(274 / 317)$ were breastfed for at least 12 months and more.

A majority, $64.7 \%$ (205/317) of the mothers to the infants in this study were known HIV positives before becoming pregnant and more than half, 57.1\% (181/317) were already on ART before pregnancy. Maternal CD4 was documented for about two-thirds of the mothers, with an overall mean CD4 of 574.25(2.69) (Table 1).

\section{HIV-free survival}

The HIV-free survival rate among infants enrolled for comprehensive care within the recommended 2 months of age in the current study, depicted by the proportion of infants discharged HIV-negative at 18 months was $94.6 \%$ $(300 / 317)$ and this was calculated only among infants with complete data.

The overall HIV infection and mortality rates in the current study were $2.5 \%(8 / 317)$ and $2.8 \%(9 / 317)$ respectively across the two facilities.

\section{Factors associated with HIV-free survival}

There was a statistically significant likelihood of a better outcome (being discharged HIV negative) among the female gender compared to males (97.9\% vs. 92.0\%) who in contrast were more likely to become infected or die (8.0\% vs 2.1\%), $P=0.024, \mathrm{OR}=3.98$ (95\% Cl, 1.12-14.12). The infants who received care from St. Mary's hospital 
Lacor were six and half times as likely to be discharged negative and survived, 98.6\% (140/142) compared to those enrolled at Gulu regional referral hospital, 91\% (160/175), and this was statistically significant, $P=0.005$, OR=6.56 (95\% Cl, 1.48-29.20).

There was no significant difference in the mean age of enrolment of the infants discharged negative (1.57, SD 0.23 ) and that of the infants who either became HIV infected or died (1.59, SD 0.20), P-value 0.685, Fstatistics=1.65. The rest of the infants' characteristics did not also have a significant association with the outcome. Likewise, none of the maternal characteristics considered in the current study was significantly associated with their infants' outcome and HIV-free survival (Table 2)

In the multivariate analysis using logistic regression, gender and treatment facility were the only independent predictors of HIV free survival. Male gender was associated with a $77 \%$ less likelihood of an HIV-free survival while being enrolled and followed up at Gulu regional referral hospital was associated with an $88 \%$ less likelihood of HIV-free survival, and these were statistically significant, $P$-values 0.28 and 0.008 respectively. The other factors in the model that were not statistically significant were NVP prophylaxis timing of maternal ART (Table 3)

\section{Discussion}

This study was carried out to evaluate the HIV-free survival rate at 18 months and associated factors among HIV exposed infants enrolled and followed up in two tertiary health facilities in Gulu district, northern Uganda under the PMTCT option B+ program.

\section{HIV-free survival}

The overall HIV-free survival rate in the current study among exposed infants enrolled within the recommended first 2 months of life was 94.6\%, while 5.4\% of the infants were either HIV-infected (2.5\%) or died 2.8\%) (Figure 2). This was calculated only among infants with complete data. Nonetheless, this highlights the impact of the current effort to eliminate mother-to-child transmission of HIV implemented initially as PMTCT option B+, and now as test and treat or treat all (mothers tested and if HIV positive, initiated on lifelong ART regardless of CD4 count or clinical stage, and infants were given ARV prophylaxis from birth for 6 or 12 weeks depending on risk classification while mothers are encouraged to exclusively breastfeed for at least 6 months). The 18-months HIVfree survival rate in the study setting only marginally falls short of the $>95 \%$ HIV-free survival rate recommended among breastfeeding populations, and is lower than the $95.9 \%$ HIV-free survival rate reported in a communitybased survey in Swaziland (13). However, the rate in the current study compares well with the 93.2\% 24-month HIV-free survival under similar Option B+ program in Rwanda (14) and is higher than the pooled estimates of 89.8\% and $85.8 \%$ for 12-month and 24-month HIV-free survival respectively as reported by Chikhungu et al (2016) in a systematic review of 18 studies evaluating HIV-free survival among breastfed infants of HIV positive women on ART (15). The above differences could largely be explained by the differences in the duration of breastfeeding and maternal ART; timing of measurements of HIV-free survival (12 months vs 18 months vs 24 months), and the guidelines or PMTCT approaches used in the different studies. Notwithstanding, these findings seem to lay credence to the effectiveness of option B+ over the earlier approaches towards the elimination of mother-to-child transmission (eMTCT) of HIV among breastfeeding population in developing countries. 
The mortality rate of $2.8 \%$ in the current study was generally low but higher than the $1.1 \%$ reported in Mma Bana trial in Botswana (16), a difference possibly explained by the fact that infants in the Mma Bana trial breastfed for a shorter duration of 6 months (median 5.8 months).

\section{Factors associated with HIV-free survival}

There was a significantly higher HIV-free survival rate for infants enrolled in Lacor (a private not for profit faithbased facility) as compared to those enrolled in GRRH (a public health facility), and this was statistically significant $(\mathrm{OR}=6.56)$. The treatment facility also remained a statistically significant predictor of HIV-free survival on multivariate analysis with an $88 \%$ increase in HIV-free survival rate for infants enrolled in Lacor as compared to those enrolled in GRRH (Table 3). We supposed this could relate to the fact that infants followed up in Lacor hospital were more likely to be enrolled slightly earlier into care (mean age 1.55[SD 0.26]) - a factor positively associated with HIV-free survival, compared to those in GRRH (mean age 1.58[SD 0.20]). This is valid and is in accord with the WHO recommendation for early infant diagnosis and a finding by Berhan et al (2014) in Ethiopia where infants with delayed DNA PCR tests had a $30 \%$ excess risk of mother-to-child transmission of HIV compared to those tested early (17). However, this difference could be as a result of several factors - both infant/caregiver factors as well as healthcare factors which were beyond the scope of this study to explore.

Similarly, there was a statistically significant likelihood of being discharged HIV negative and survival among the female gender compared to males, who in contrast were more likely to become infected or die. In the multivariate analysis, there was a $77 \%$ increased chance of HIV-free survival if an infant was of a female compared to being male. The difference in outcome among HIV exposed infants by gender has not been well explained in previous studies. However, one plausible view could relate to the fact that an increased risk of morbidity and mortality among young males, in general, has long been advanced, albeit with no well understood scientific explanations $(18,19)$. In the context of HIV therefore, this could translate to faster disease progression in males compared to female infants.

The overall duration of breastfeeding did not significantly affect the HIV-free survival in the current study. This finding compares well with that reported by Alvarez-Uria et al (2012) in India (20) and Peltier et al (2009) in Rwanda (21) where there was no significant difference in HIV-free survival with breastfeeding status, though HIVfree survival was significantly higher among breastfed than formula-fed children in the two reports. Besides, the Rwandan study estimated HIV-free survival at an earlier time interval of 9 months during a different approach to PMTCT and the infants were breastfed for only 5-6 months (exclusive breastfeeding) followed by rapid weaning. In contrast, Mekonnen A, et al (2017) reported a significantly lower 18 months cumulative probability of HIV-free survival in the breast-fed infants and young children (84\%) than formula-fed counterparts (97\%) (22).

While the majority (90.5\%) of the infants were reported to have had exclusive breastfeeding (EBF), less than one third (29.3\%) were exclusively breastfed for at least 6 months as recommended by the national guidelines. The overall rate of exclusive breastfeeding in this study though compares well with that reported by Okafor et al (2014) in Nigeria of $91.8 \%$ (23). However, by implication, the fact that only very few mothers (29.3\%) in the study setting are exclusively breastfeeding their infants adequately for at least 6 months should be of concern since it could be a precursor of prevailing misinformation and inherent negative perception about breastfeeding in the context of HIV which calls for more awareness and counselling. By and large, exclusive breastfeeding for at least 6 months in the current study was protective and associated with $55 \%$ increased chance of HIV-free survival 
compared to $\mathrm{EBF}$ for $<6$ months, although this was not statistically significant, $\mathrm{OR}=0.45$ (Table 2 ). The above findings could be attributed to the fact that regardless of breastfeeding duration, maternal ART and timely infant ARV prophylaxis are critical factors in the prevention of mother-to-child transmission of HIV, in line with the current guidelines.

In the current study, the timing of maternal HIV diagnosis and ART initiation did not statistically significantly influence HIV-free survival. Infants of mothers diagnosed before pregnancy were $12 \%$ less likely to have an HIVfree survival, while those born to mothers who initiated ART before pregnancy were $37 \%$ less likely to have an HIV-free survival. However, the documented HIV transmission rate was lower among infants of mothers initiated on ART before pregnancy (1.7\%) compared to the rate among infants of mothers initiated during pregnancy (3.9\%). This finding is in keeping with that reported in a Cameroonian study in which the 12-month HIV transmission rate where $51 \%$ of women were receiving ART before pregnancy was low at $1.2 \%$ (24), and also compares relatively well with that reported in a study by Hoffman et al (2010) where MTCT rate was lower in women who were on HAART before pregnancy compared to women who initiated HAART during pregnancy (25). The similar benefit of prior ART maternal use was reported by Oluwayemi et al (2015) in Nigeria where the risk of transmission was significantly lower among babies whose mothers commenced HAART before pregnancy (3.4\%) compared to those whose mothers initiated HAART during pregnancy (5.4\%) (26). The above results could be explained by the fact that one of the hypothesized benefits of lifelong ART is protection against HIV transmission in subsequent pregnancies, resulting from greater chances of virologic suppression, as was also suggested by Gill et al (2017) in a Rwandan study where the substantial proportion of women on ART before pregnancy with suppressed viral load (VL) was thought to have contributed to the high effectiveness of PMTCT (14).

Likewise, available evidences also point to the fact that long duration of ART may be associated with high viral load (VL) or viral rebound postpartum which could be associated with increased risk of mother-to-child transmission $(14,27,28)$, supporting the importance of $V L$ monitoring during pregnancy and breastfeeding, and continued adherence counselling (14). In the current study, data on maternal viral load suppression was very limited (VL was not widely available in Uganda within the period for which data was collected for this study), making comparison of HIV-free survival based on maternal VL impossible.

\section{Limitations of the Study}

Being a retrospective study with the use of secondary data, the information retrieved was incomplete for some infants. Some of the maternal potential factors for vertical transmission could not be exhaustively explored in the study because they were lacking. Secondly, exclusion of cases lost to follow up and those with the unknown outcome or missing data is likely to have created a selection bias, affecting the accuracy of the estimates of survival and therefore the generalizability (external validity) of the study findings. Our estimates may, therefore, be an overestimate or underestimate since some infants with missing data and undocumented outcome were not considered. Our results could, therefore, be skewed since for instance, it is likely that those missing an outcome more frequently died than remained HIV-free and survived

\section{Conclusion}


Our study estimates that the HIV-free survival rate in the study setting for breastfed infants enrolled by 2 months of age was $94.6 \%$, below the WHO goal of $>95 \%$ HIV-free rate in breastfeeding population for virtual elimination of mother-to-child transmission of HIV.The overall duration of breastfeeding did not significantly affect HIV-free survival. However, a further prospective study would be necessary to assess a more accurate HIV-free survival rate given the limitations of retrospective data.

\section{Declarations}

\section{Ethics approval and consent to participate}

The study was approved by the Gulu University Research and Ethics Committee (GUREC) and permission to conduct the study and access records was granted by the respective research committees of the respective hospitals. Being a retrospective study the patient consent was deemed unnecessary. However, the identity of the participants was kept confidential by using participants' codes and not names.

\section{Consent for publication}

Participants' consent to publish this manuscript was not applicable since the manuscript does not contain any participants' data.

\section{Availability of data and materials}

The dataset supporting the conclusions of this article is available on request to the corresponding author.

\section{Competing interests}

The authors declare that they have no competing interests.

\section{Funding}

This study relied on the investigators' resources in terms of time and efforts and did not receive any external funding.

\section{Authors' contributions}

All authors contributed to the design of the study. Al, KC, AP and MS participated in data collection and entry. NR performed statistical analysis. All authors participated in drafting the manuscript. All authors read and approved the final manuscript.

\section{Acknowledgements}

We are thankful to the staffs of Gulu regional referral hospital and St. Mary's hospital Lacor ART clinics whose support made this study possible. A special tribute goes to the participants whose data contributed to the knowledge generated by this study.

\section{Abbreviations}


WHO: World Health Organization; HIV: Human Immunodeficiency virus; ART: Antiretroviral Therapy; ARV:

Antiretroviral; GRRH: Gulu Regional Referral Hospital; MTCT: Mother-to-child transmission; PMTCT: Prevention of mother-to-child transmission; eMTCT: Elimination of mother-to-child transmission; AIDS: Acquired Immunodeficiency Syndrome; EBF: Exclusive Breastfeeding; CF: Complementary Feeding; 3TC: Lamivudine; TDF: tenofovir disoproxil fumarate; EFV: Efavirenz; DNA: Deoxyribonucleic Acid; PCR: Polymerase Chain Reaction; DBS: Dry Blood Spot; EID: Early Infant Diagnosis; HAART: Highly Active Antiretroviral Therapy; VL: Viral Load.

\section{References}

1. UNAIDS data 2018. available at: https://www.unaids.org/en/resources/documents/2018/unaids-data-2018.

2. Uganda Population-based HIV Impact Assessment (UPHIA 2016-2017), August 2017; available from: https://www.afro.who.int/sites/default/files/2017-08/UPHIA\%20Uganda\%20factsheet.pdf.

3. WHO, UNICEF, UNAIDS, UNFPA. HIV Transmission Through Breastfeeding A Review of Available Evidence An Update from 2001 to 2007; available from: www.who.int/nutrition/topics/Paper_5_Infant_Feeding_bangkok.pdf.

4. UNAIDS: Global Report: UNAIDS Report on the Global AIDS Epidemic. Geneva: 2012. Available from: http://www.unaids.org/en/media/unaids/contentassets/documents/epidemiology/2012/gr2012/20121120 unaids_global_report_2012_with_annexes_en.pdf.

5. World Health Organization, WHO antiretroviral therapy for infants and children. Report of the WHO Technical Reference Group, Paediatric HIV/ART Care Guideline Group meeting. Geneva: World Health Organization; 2008.

6. World Health Organization. Consolidated guidelines on the use of antiretroviral drugs for treating and preventing HIV infection. Geneva: World Health Organization; 2013.

7. Ministry of Health, Uganda. Consolidated Guidelines for Prevention and Treatment of HIV in Uganda, December 2016. Available from: https://aidsfree.usaid.gov/sites/default/files/uganda_hiv_gl_2016.pdf.

8. World Health Organization. Guidelines on When to Start Antiretroviral Therapy and on Pre-Exposure Prophylaxis for HIV. Geneva, Switzerland: World Health Organization; 2015. Available from: http://www.who.int/hiv/pub/guidelines/earlyrelease-arv/en/.

9. Uganda Bureau of Statistics (UBOS). Findings from the national population and housing census 2014. Available from; https://www.ubos.org/.../03_20182014.

10. Leslie Kish. Survey Sampling. John Wiley and Sonsa. New York. 1965:59-60.

11. Ministry of Health, Uganda. Addendum to the National Antiretroviral Treatment Guidelines, December 2013.

12. World Health Organization. Guideline Updates on HIV and infant feeding: the duration of breastfeeding, and support from health services to improve feeding practices among mothers living with HIV. Geneva: World Health Organization; 2016.

13. Caspian Chouraya, Rhoderick Machekano, Simangele Mthethwa, Krysia Lindan, Munamato Mirira, Kwashie Kudiabor, et al. Mother-to-Child Transmission of HIV and HIV-Free Survival in Swaziland: A Community-Based Household Survey. AIDS and Behavior 2018;22:S105-S13.

14. Gill MM, Hoffman HJ, Ndatimana D, Mugwaneza P, Guay L, Ndayisaba GF, et al. 24-month HIV-free survival among infants born to HIV-positive women enrolled in Option B+ program in Kigali, Rwanda. The Kabeho Study. Medicine. 2017;96:51.

Page 10/15 
15. Chikhungu LC, Bispo S, Rollins N, Siegfried N, Newell ML. HIV-free survival at 12-24 months in breastfed infants of HIV-infected women on antiretroviral treatment. Tropical Med Int Health. 2016;21(7):820-8.

16. Shapiro RL, Kitch D, Ogwu A, Hughes MD, Lockman S, Powis K, et al. HIV transmission and 24-month survival in a randomized trial of HAART to prevent MTCT during pregnancy and breastfeeding in Botswana (The Mma Bana Study). AIDS. 2013;27(12):1911-20.

17. Berhan Z, Abebe F, Gedefaw M, Tesfa M, Assefa M, Tafere Y. Risk of HIV and associated factors among infants born to HIV positive women in Amhara region, Ethiopia: a facility-based retrospective study. BMC Research Notes. 2014;7:876: http://www.biomedcentral.com/1756-0500/7/876.

18. Gissler M, Ja“rvelin M-R, Louhiala P, Hemminki E. Boys have more health problems in childhood than girls: follow-up of the 1987 Finnish birth cohort. Acta Pñdiatr 1999;88:310 4 .

19. van den Bosch W, Huygen F, van den Hoogen $H$, van Weel C. Morbidity in early childhood: differences between girls and boys under 10 years old. British Journal of General Practice. 1992;42:366-9.

20. Alvarez-Uria G, Midde M, Pakam R, Bachu L, Naik PK. Effect of Formula Feeding and Breastfeeding on Child Growth, Infant Mortality, and HIV Transmission in Children Born to HIV-Infected Pregnant Women Who Received Triple Antiretroviral Therapy in a Resource-Limited Setting: Data from an HIV Cohort Study in India. ISRN pediatrics 2012;2012:763591.

21. Peltier CA, Ndayisaba GF, Lepage P, van Griensven J, Leroy V, Pharm CO, et al. Breastfeeding with maternal antiretroviral therapy or formula feeding to prevent HIV postnatal mother-to-child transmission in Rwanda. AIDS (London, England) 2009;23(18):2415-23.

22. Mekonnen Assefa, Alemayehu Worku, Aman Yesuf. HIV-free survival and morbidity among breast-fed and formula-fed infants and young children in prevention of MTCT of HIV program in Addis Ababa, Ethiopia, 2014. Asian Pac J Trop Dis. 2017;7(4):225-32.

23. Okafor II, Ugwu EO, Obi SN, Odugu BU. Virtual elimination of mother-to-child transmission of human immunodeficiency virus in mothers on highly active antiretroviral therapy in Enugu, South-Eastern Nigeria. Ann Med Health Sci Res. 2014;4:615-8.

24. Altan AM, Taafo F, Fopa F, et al. An assessment of Option B implementation for the prevention of mother to child transmission in Dschang, Cameroon: results from the DREAM (Drug Resource Enhancement against AIDS and Malnutrition) cohort. Pan Afr Med J. 2016;23:72.

25. Hoffman R, Black V, Technau K, van der Merwe KJ, Currier J, Coovadia A, et al. Effects of Highly Active Antiretroviral Therapy Duration and Regimen on Risk for Mother-to-Child Transmission of HIV in Johannesburg, South Africa. J Acquir Immune Defic Syndr. 2010;54(1):35-41.

DOI:10.1097/QAI.0b013e3181cf9979.

26. Oluwayemi IO, Olatunya SO, Ogundare EO. PCR Results and PMTCT Treatment Outcomes among HIVExposed Infants in a Tertiary Hospital in Nigeria, 2010-2014. International Journal of MCH and AIDS 2015;3(2):168-73.

27. Gill MM, Hoffman HJ, Bobrow EA, et al. Detectable viral load in late pregnancy among women in the Rwanda option B+ PMTCT program: enrollment results from the Kabeho study. PLoS One 2016;11:e0168671.

28. Huntington $S$, Thorne $C$, Newell $M-L$, et al. The risk of viral rebound in the year after delivery in women remaining on antiretroviral therapy. AIDS. 2015;29:2269-78.

Page $11 / 15$ 


\section{Tables}

Table 1 Baseline characteristics of the study population ${ }^{[A 1]}$

\begin{tabular}{|c|c|c|}
\hline \multirow{2}{*}{\multicolumn{3}{|c|}{$\begin{array}{l}\text { Characteristics } \\
\text { Maternal baseline characteristics }\end{array}$}} \\
\hline & & \\
\hline Mean CD4 count & \multicolumn{2}{|l|}{$574.25(2.69) *$} \\
\hline §CD4 Count: & & \\
\hline$\geq 500$ cells $/ \mathrm{m} 3$ & 116 & 36.6 \\
\hline$<500$ cells $/ \mathrm{m} 3$ & 95 & 30.0 \\
\hline Unknown & 106 & 33.4 \\
\hline \multicolumn{3}{|l|}{ HIV Diagnosis: } \\
\hline Before pregnancy & 205 & 64.7 \\
\hline During pregnancy & 105 & 33.1 \\
\hline Postpartum & 7 & 2.2 \\
\hline \multicolumn{3}{|l|}{ ART initiation: } \\
\hline Before pregnancy & 181 & 57.1 \\
\hline During pregnancy & 128 & 40.4 \\
\hline Postpartum & 8 & 2.5 \\
\hline \multicolumn{3}{|l|}{ Infant baseline characteristics } \\
\hline Mean age (months) & $1.57(0.23)^{*}$ & \\
\hline \multicolumn{3}{|l|}{ Sex: } \\
\hline Male & 176 & 55.5 \\
\hline Female & 141 & 44.5 \\
\hline \multicolumn{3}{|l|}{ Treatment facility } \\
\hline Lacor & 142 & 44.8 \\
\hline GRRH¥ & 175 & 55.2 \\
\hline \multicolumn{3}{|l|}{ NVP prophylaxis: } \\
\hline Yes & 315 & 99.4 \\
\hline No & 2 & 0.6 \\
\hline
\end{tabular}

* Mean and Standard deviation, ¥Gulu regional referral hospital

Table 2 Infants' and maternal characteristics associated with HIV-free survival ${ }_{[\mathrm{A} 1]}$ 


\begin{tabular}{|c|c|c|c|c|c|}
\hline & $\begin{array}{l}\text { HIV Free } \\
\mathrm{N}=342(\%)\end{array}$ & $\begin{array}{l}\text { Infected/Died } \\
\mathrm{N}=23(\%)\end{array}$ & $\mathrm{OR}^{\alpha}$ & $95 \% \mathrm{CI}$ & $P$-value \\
\hline \multicolumn{6}{|l|}{ Infants' baseline characteristics } \\
\hline ФMean age (months) & $1.57(0.23)$ & $1.59(0.20)$ & \multirow{2}{*}{\multicolumn{2}{|c|}{$0.165^{\Pi}$}} & 0.685 \\
\hline \multicolumn{4}{|l|}{ Gender: } & & \\
\hline Female & 138 (97.9) & $3(2.1)$ & \multirow[t]{2}{*}{3.98} & \multirow[t]{2}{*}{$1.12-14.12$} & \multirow[t]{2}{*}{$0.024 *$} \\
\hline Male & $162(92.0)$ & $14(8.0)$ & & & \\
\hline \multicolumn{6}{|l|}{ Treatment facility: } \\
\hline Lacor & $140(98.6)$ & $2(1.4)$ & \multirow[t]{2}{*}{6.56} & \multirow[t]{2}{*}{$1.48-29.20$} & \multirow[t]{2}{*}{$0.005^{*}$} \\
\hline GRRH & $160(91.4)$ & $15(8.4)$ & & & \\
\hline \multicolumn{6}{|l|}{ Infants' Interventions } \\
\hline \multicolumn{6}{|l|}{ NVP prophylaxis: } \\
\hline Yes & 299 (94.9) & $16(5.1)$ & \multirow{2}{*}{18.69} & \multirow[t]{2}{*}{$1.12-312.60$} & \multirow[t]{2}{*}{0.105} \\
\hline No & $1(50.0)$ & $1(50.0)$ & & & \\
\hline \multicolumn{6}{|l|}{ §Duration of EBF: } \\
\hline$<6$ months & $185(95.4)$ & $9(4.6)$ & \multirow{2}{*}{0.45} & \multirow[t]{2}{*}{$0.10-2.14$} & \multirow[t]{2}{*}{0.512} \\
\hline At least $6 \mathrm{mo}$ & $91(97.8)$ & $2(2.2)$ & & & \\
\hline \multicolumn{6}{|l|}{ §Total B/feeding: } \\
\hline$\square 12$ months & $13(100)$ & $0(0.0)$ & \multirow{2}{*}{1.02} & \multirow[t]{2}{*}{$1.00-1.04$} & \multirow[t]{2}{*}{1.000} \\
\hline$\geq 12$ months & $269(98.2)$ & $5(1.8)$ & & & \\
\hline \multicolumn{6}{|l|}{ Maternal characteristics } \\
\hline \multicolumn{6}{|l|}{ ¥HIV Diagnosis: } \\
\hline Before pregnancy & $194(94.6)$ & $11(5.4)$ & \multirow[t]{2}{*}{0.88} & \multirow[t]{2}{*}{$0.30-2.61$} & \multirow[t]{2}{*}{0.820} \\
\hline During pregnancy & $100(95.2)$ & $6(4.8)$ & & & \\
\hline \multicolumn{6}{|l|}{$\S \mathrm{CD} 4$ Count } \\
\hline$\geq 500$ cells $/ \mathrm{m} 3$ & $110(94.8)$ & $6(5.2)$ & \multirow[t]{2}{*}{1.02} & \multirow[t]{2}{*}{$0.30-3.45$} & \multirow[t]{2}{*}{1.000} \\
\hline$<500$ cells $/ \mathrm{m} 3$ & $90(94.7)$ & $5(5.3)$ & & & \\
\hline$¥ A R T$ initiation: & & & & & \\
\hline Before pregnancy & 170 (93.9) & $11(6.1)$ & 0.63 & $0.21-1.85$ & 0.396 \\
\hline During pregnancy & $123(96.1)$ & $5(3.9)$ & & & \\
\hline
\end{tabular}

${ }^{\alpha}$ Unadjusted Odds ratio, ${ }^{\Pi} F$ statistic, 9 Mean (SD), *P-value significant (<0.05); §Only those with documented NVP timing, duration of EBF and the total duration of breastfeeding were considered. ¥Only those diagnosed for HIV and initiated on ART before pregnancy and during pregnancy, excluding those diagnosed and initiated on ART after delivery.

Table 3 Logistic regression for factors independently predicting HIV-free survival ${ }^{\S}$

\begin{tabular}{llll}
\hline Characteristics & AOR & 95\% CI & p-value \\
\hline Gender & 0.23 & $0.06-0.85$ & $\mathbf{0 . 0 2 8 *}$ \\
Treatment facility & 0.12 & $0.02-0.57$ & $\mathbf{0 . 0 0 8 *}$ \\
NVP prophylaxis & 0.07 & $0.00-1.90$ & 0.114 \\
Timing of maternal ART & 2.32 & $0.74-7.32$ & 0.149 \\
\hline
\end{tabular}

* $P$-value significant (<0.05), AOR=Adjusted Odds ratio, $C I=95 \%$ confidence interval $\S$ Included in the multivariable analysis were all variables with $p$-value less than 0.2 and those with scientific plausibility with $p$-value $<0.5$. This included gender, treatment facility, NVP prophylaxis, and timing of maternal ART. 


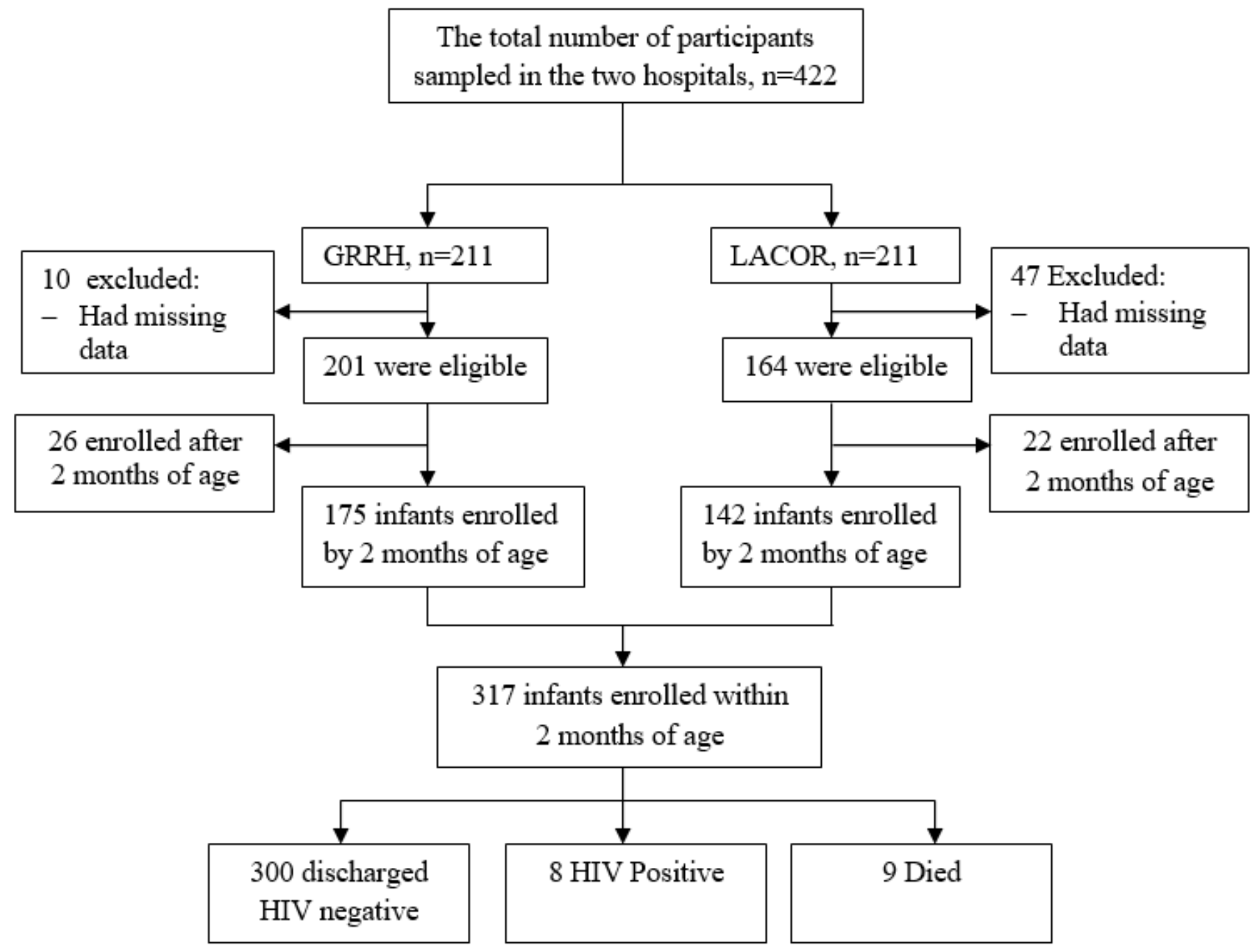

Figure 1

Study profile 


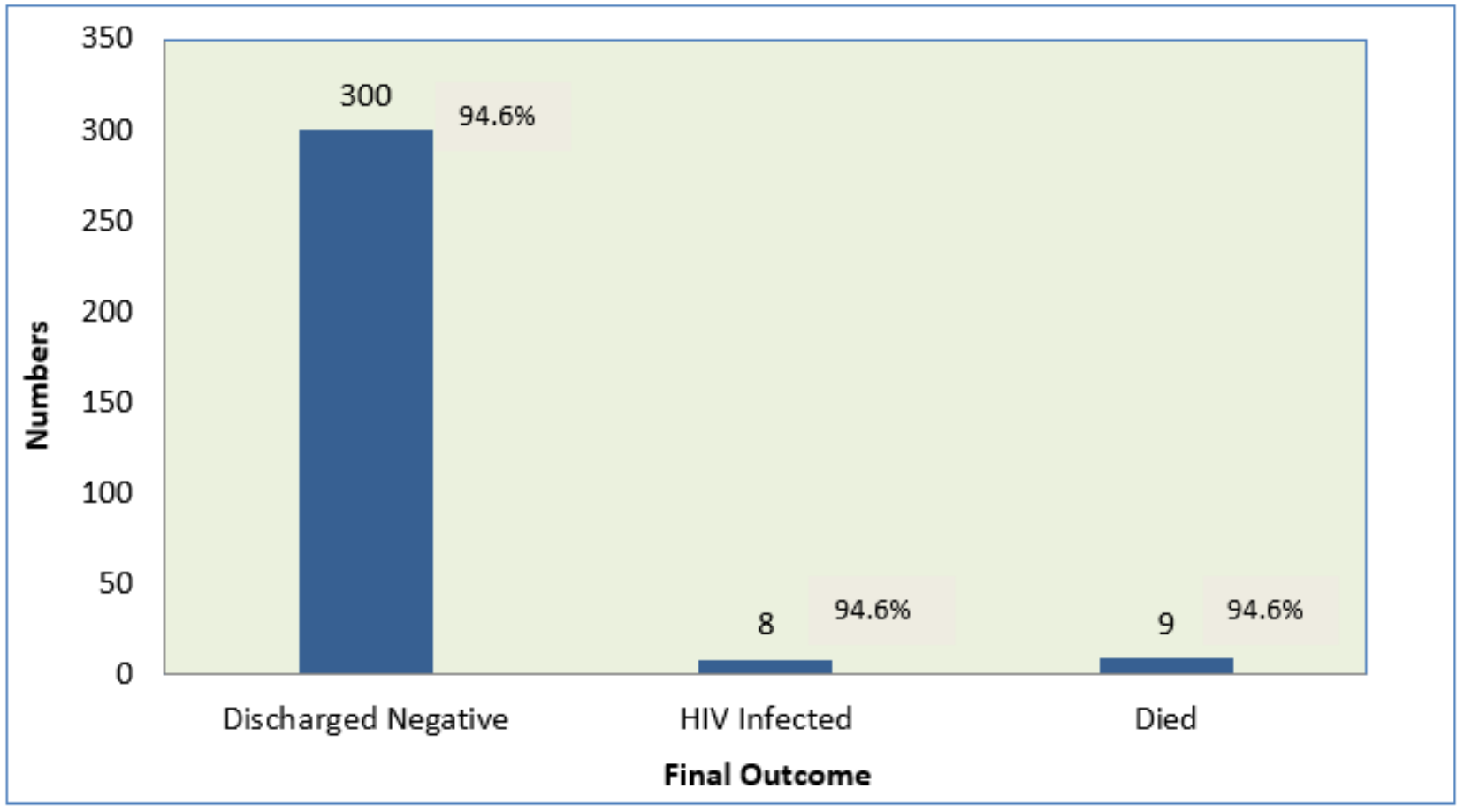

Figure 2

Final outcome at 18 months among infants enrolled by $0-2$ months of age. 